\title{
Switching Cost Minimization in the IEEE 802.16e Mobile WiMAX Sleep Mode Operation
}

\author{
Gary K. W. Wong \\ Department of Computer \\ Science and Engineering, The \\ Hong Kong University of \\ Science and Technology \\ Clear Water Bay \\ Kowloon, Hong Kong \\ wongk@cse.ust.hk
}

\author{
Qian Zhang \\ Department of Computer \\ Science and Engineering, The \\ Hong Kong University of \\ Science and Technology \\ Clear Water Bay \\ Kowloon, Hong Kong \\ qianzh@cse.ust.hk
}

\author{
Danny H. K. Tsang \\ Department of Electronic and \\ Computer Engineering, The \\ Hong Kong University of \\ Science and Technology \\ Clear Water Bay \\ Kowloon, Hong Kong \\ eetsang@ece.ust.hk
}

\begin{abstract}
To prolong the battery lifetime, it is important to continue designing a better energy efficient mechanism for different mobile technologies. Most of the existing works on the IEEE 802.16e sleep mode operation focus on the decision making before a mobile station switching to sleep mode state. The correlation of the decision is mainly on when and how to sleep based on the traffic demands. After the mobile station is switched to sleep mode, the deactivation of it mainly depends on new incoming traffic regardless of the actual amount. Truly, frequent switching can increase the energy cost on the mobile station, which can significantly reduce the battery lifetime. To minimize the switching frequency, we propose a novel approach to resolve this issue by making a heuristic decision during the listening interval. With this aim, we propose a real-time heuristic algorithm, WAKSLP_DECISION, to accommodate our target. Three main decision criteria are analyzed and designed, namely the probability of buffer overflow, expected delay violation, and battery lifetime expiry, to achieve our goal. We verify the energy consumption performance with simulation experiments to validate our proposed scheme. The result shows that our scheme performs $25 \%$ to $30 \%$ better compared with the original standard in terms of energy consumption. We believe this algorithm is practical and implementable without changing the original standard, which can contribute both in the research community and industrial development.
\end{abstract}

\section{Categories and Subject Descriptors}

C.2.1 [Computer-Communication Networks]: Network Architecture and Design-Network communications, Wireless communication, Distributed networks

Permission to make digital or hard copies of all or part of this work for personal or classroom use is granted without fee provided that copies are not made or distributed for profit or commercial advantage and that copies bear this notice and the full citation on the first page. To copy otherwise, to republish, to post on servers or to redistribute to lists, requires prior specific permission and/or a fee.

IWCMC '09 June 21 - 24, 2009, Leipzig, Germany

Copyright 2009 ACM 978-1-60558-569-7/09/06 ...\$5.00.

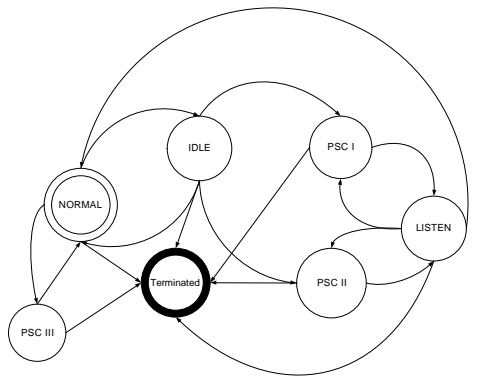

Figure 1: State-transition diagram for Mobile WiMAX Sleep Mode Operation

\section{General Terms}

Algorithms, Designs, Performance

\section{Keywords}

Energy-efficiency wireless, sleep mode, 802.16e, power management

\section{INTRODUCTION}

Broadband wireless stands at the confluence of two of the most significant and remarkable growth stories of the telecommunication industry in recent years. Due to the enormous impact of this technology, the world has called for higher expectation and improvement for the existing working systems to provide with various types of services.

For the past few years, we have witnessed the convergence of the IEEE standard for broadband wireless, IEEE 802.16 [1]. In the industry, this standard has been adopted to be the global standard for metropolitan area network with wireless connectivity. The popularity of this standard is indeed undeniable. In the original WiMAX proposal, the mobility issue was not fully discussed and supported. Later on in the IEEE 802.16e [2] amendment, mobility is finally supported and standardized to realize the mobile applications.

Since the release of this standard, a few significant research studies have been dedicated for the power management. One suggested solution to save the power usage of a mobile WiMAX device is to implement the sleep mode feature. When the device is idle, sleep mode will be activated so as to minimize the power consumption by using the min- 
imal power to maintain the running system. Most of these research works focus on when to sleep and how to sleep. Yet, none of those works discuss the possibility of making an optimal decision of when to wake up. In this paper, we contribute our effort in the investigation of the benefits in considering when to wake up for better energy efficiency.

No doubt, the probability of a large packets accumulation at base station becomes low when traffic is not heavy. In fact, frequent switching from sleep mode to normal mode can result in excessive power consumption and should not be neglected [6]. If a mobile station switches back and forth regardless of the amount of data to be received, unnecessary energy could be wasted. This problem has not been carefully addressed among all the existing works. Thus, we propose a novel scheme to minimize the switching frequency by introducing three basic criteria to decide if a mobile station should wake up for transmission. The criteria of permitting the mobile station to remain in the sleep mode are achieved when the three proposed Quality of Service (QoS) requirements are filled, namely the buffer overflow probability, expected delay violation, and battery lifetime expiry. We believe that our contribution is the first successful work in addressing the issue of when to wake up for better energy efficiency.

The rest of this paper will be organized as follows. In Section II, We give a brief discussion about the research works related to the power management and energy saving mechanism. In Section III, we provide with a brief discussion about the background of sleep mode in the IEEE 802.16e. In Section IV, the framework of the algorithm and the design are presented in details. In Section V, we explain our methodology and simulation parameters settings in the next section. Afterward, we present our simulation results with discussion. In Section VI, we conclude our paper with a summary.

\section{RELATED WORK}

In the IEEE 802.16e, three Power Saving Classes (PSCs) are available to sustain different types of traffics in the network [2]. Note that each sleep period can be constant and varying, depending on the types of sleep modes. Regardless of which PSC a mobile subscriber (MSS) picks, the three PSCs share the same procedural operation. The statetransition diagram in Figure 1 illustrates the transitions between each state.

To fully support mobility, issues related to power saving are in the top priority. Several significant research studies have been dedicated for the power saving mechanism, particularly the sleep mode operation. In [3] and [4], the authors propose an analytical model of PSC type I to capture the energy consumption levels including both incoming and outgoing frames. Similar to all other proposed designs, they only consider type I. In [5] and [6], the authors include type I and type II under their investigation. In [5], the authors formulates the system as an embedded Markov Chain model under timeout scheme, while in [6], the authors provide a method to determine the optimal selection of PSC by formulating the problem using the Markov Decision Process (MDP) model to solve it by Policy Optimization. Different from the original standard, authors in [7] propose an adaptively scheme to adjust the sleeping windows based on the current system traffic demands. In [8], initial and final sleeping windows are adaptive adjusted according to the current

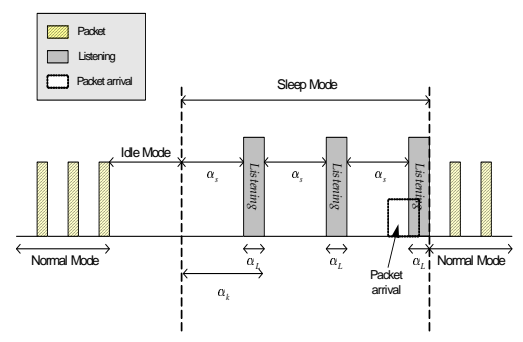

Figure 2: Mobile WiMAX Sleep Mode Operation with Fixed Sleeping Window Size (Type II)

remaining energy level.

Indeed, most of the previous research works are somehow similar in terms of the optimality of decision making. They seem to merely focus on when a mobile station should switch to sleep mode and how by choosing an appropriate sleeping behavior from the standardized categories. Yet, they simply neglect the possibility of making decision during the activation of sleep mode. Thus, we propose a novel scheme to allow the MSS to decide heuristically if it should wake up every time when a positive traffic indicator is received during the listening interval.

\section{IEEE 802.16E SLEEP MODE OPERATION}

As we have discussed, sleep mode is a state when a MSS is absent from the serving BS for a pre-negotiated periods of time. During this period, the MSS will not receive any downlink traffic. All the packets for the MSS will be buffered in the BS until the MSS switches to normal mode again. Regardless of which PSC type the MSS picks, the procedures are similar. When a MSS decides to put itself into sleep mode, it sends a MOB_SLP-REQ message to the serving BS to request for the approval to switch. In the message, the MSS specifies some important parameters required for different PSCs. Once the request is approved, the BS will send back the response with a MOB_SLP-RSP message. After the message is received, the MSS begins to sleep according to the choice of the PSC. During the sleep mode duration, the MSS wakes up for a short period of time called listening interval. During this interval, the BS will send a message called MOB_TRF-IND as a traffic indication to signify if any traffic has been buffered in the BS while in the sleep mode. If the MSS receives a positive indicator, it will wake up and activate all the necessary components to begin receiving the packets. If the negative indicator is received, it will continue to remain in the sleep mode until the next positive traffic indication. A simple illustration of PSC type II can be referred to Fig. 2 .

In short, these three types of PSCs differ by their parameter sets, procedures of activation and deactivation, and policies of MSS availability for data transmission. For PSC type I, the sleeping period for each stage can be computed as follows:

$$
\alpha_{k}=\alpha_{0} \cdot 2^{k}+\alpha_{L}, \forall k=\{1,2, \ldots, w\}
$$

Based on Equation 1, the total sleeping period is a combination of a sleeping window followed by a listening interval. Note that if the traffic indicator is negative, the sleeping period is doubled each time until the maximum sleeping stage is achieved. This exponential growth is suitable for those 


\begin{tabular}{|c|l|}
\hline Symbols & Descriptions \\
\hline$\lambda$ & Packet arrival rate at the base station buffer \\
\hline$\mu$ & Service rate at the mobile station \\
\hline$w$ & Maximum sleeping stage \\
\hline$\alpha_{0}$ & Initial sleeping window size (for Type I only) \\
\hline$\alpha_{s}$ & Constant sleeping window size (for Type II and III only) \\
\hline$\alpha_{L}$ & Constant time period for a listening interval \\
\hline$\alpha_{k}$ & Total sleeping period of stage $k$ \\
\hline$L_{B S}$ & Buffer size at the base station \\
\hline$i$ & Current buffer occupancy at the base station \\
\hline$l_{i}\left(\alpha_{k}\right)$ & Expected buffer occupancy after a period of $\alpha_{k}$ \\
\hline$B$ & Upper bound of buffer overflow probability set by the mobile station \\
\hline$\tau$ & Packet arrival time relative to the beginning time of the next sleeping window \\
\hline$\rho$ & Traffic intensity of the communication system \\
\hline$D$ & Upper bound of expected delay violation set by the mobile station \\
\hline$\mu^{\prime}$ & Energy consumption rate during the service of packets \\
\hline$E_{r e m a i n}$ & Energy remaining in the mobile station \\
\hline
\end{tabular}

traffic that have low probability of new arrival after the station enters the sleep mode. For PSC type II, the sleeping period for each stage can be computed as follows:

$$
\alpha_{k}=\alpha_{s}+\alpha_{L}, \forall k=\{1,2, \ldots, \infty\}
$$

As we can see, the only difference compared to Equation 1 is the constant sleeping window for each stage. If the traffic is not detected at the listening interval, the mobile station continues to sleep with the same period of time as before. For PSC type III, the sleeping period for each stage can be computed as follows:

$$
\alpha_{k}=\alpha_{s}
$$

Different from the other two types, this does not contain a listening interval due to the original purpose of this power saving class. After the mobile station sleeps for a predetermined period, it wakes up immediately without performing any traffic detection.

\section{FRAMEWORK OF THE ALGORITHM}

Our algorithm mainly consists of three major criteria for our heuristic decision making.

\section{Buffer Overflow Probability \\ 2. Expected Delay Violation \\ 3. Battery Lifetime Expiry}

Suppose we decide to sleep for one more period of sleeping windows size, we should consider the three criteria above. The fundamental principle of decision making starts with the first criterion. Because of the nature of WiMAX network, a dedicated finite buffer is allocated for each MSS at the BS.

\subsection{Probability of Buffer Overflow}

Our objective in this section is to develop the probability of the finite buffer overflow at the base station.

Given that the traffic arrival rate follows the Poisson distribution. Indeed, our algorithm can fit into a general traffic arrival distribution. In order to simplify our discussion, we pick one particular distribution for our analysis. With the Poisson property [9], then we immediately know that if the arrival rate is $\lambda$, the probability for $n$ numbers of packet arrival during a period of $\alpha_{k}$ would be

$$
P\left(n \text { arrival during } \alpha_{k}\right)=\frac{\left(\lambda \alpha_{k}\right)^{n} e^{-\lambda \alpha_{k}}}{n !}
$$

Suppose the finite buffer size of the base station is $L_{B S}$ with the current buffer occupancy denoted by $i$. In order to observe the buffer to be overflowed, the increase of the packets during the period of $\alpha_{k}$ must be $n \geq L_{B S}-i+1=$ $L_{B S}-(i-1)$. So, we can then derive the probability that the buffer will be overflowed as

$$
\begin{aligned}
P\left(l_{i}\left(\alpha_{k}\right)>L_{B S}\right) & =P\left(n \geq L_{B S}-(i-1)\right) \\
& =\sum_{n=L_{B S}-(i-1)}^{\infty} \frac{\left(\lambda \alpha_{k}\right)^{n} e^{-\lambda \alpha_{k}}}{n !}
\end{aligned}
$$

Thus, if the mobile station specifies the bound as $B$, then the criteria can be set to

$$
\sum_{n=L_{B S}-(i-1)}^{\infty} \frac{\left(\lambda \alpha_{k}\right)^{n} e^{-\lambda \alpha_{k}}}{n !} \leq B
$$

This will conclude the calculation of the buffer overflow probability. Note that this criterion requires a prior knowledge of the traffic rate $\lambda$ and the packet arrival distribution. In order to obtain an accurate value of $\lambda$ and the associated distribution, the mobile station is expected to have a traffic estimator and the arrival distribution detector. The discussion of different estimator and detector is out of our scope for this paper. We only need to assume that the mobile station has a way to learn those two information.

Next, we will discuss the calculation of the expected delay violation condition.

\subsection{Expected Delay Violation}

In order to stay in the sleep mode even though a positive indicator is received, a MSS should consider if packet delay requirement is violated. The meaning behind this delay criterion is to make sure that no matter when the packet arrives during the next sleeping window, the expected delay will not exceed the delay violation tolerable bound. After we receive the status of the current buffer occupancy value during the current listening interval, we actually can calculate 
and estimate all the expected delay for each packet arriving in the next period of $\alpha_{k}$. Suppose $\tau$ is the arrival time of some future packet during the next sleeping window. Based on this $\tau$, we can calculate the expected delay and observe if it violates our expected delay bound. However, it may impose a large processing delay due to the computation cost if we are to calculate the delay values for all future packets. In order to minimize the computational delay, the most efficient method is to look for the strongest condition based on the different traffic intensity.

Objective: To verify the impact of $\tau$ in the general form of the linear equation

$$
\max _{\tau}\left\{(i+\lambda \tau) \cdot \frac{1}{\mu}+\left(\alpha_{k}-\tau\right): \forall \tau \in\left[0, \alpha_{k}\right]\right\} \leq D
$$

The strongest conditions should be the following two inequalities,

$$
\begin{aligned}
\tau & =0: i \cdot \frac{1}{\mu}+\alpha_{k} \leq D \text { for } \rho \leq 1 \\
\tau & =\alpha_{k}:\left(\alpha_{k} \lambda+i\right) \cdot \frac{1}{\mu} \leq D \text { for } \rho>1
\end{aligned}
$$

where $\rho=\frac{\lambda}{\mu}$.

Theorem 1. Given $\mu, \lambda, \rho, \alpha_{k} \in\left[\mathbb{R}^{+} \cup 0\right], i \in\left[\mathbb{Z}^{+} \cup 0\right]$, $\rho=\frac{\lambda}{\mu}$, and $\tau \in E=\left[0, \alpha_{k}\right]$. Let $d: E \rightarrow \mathbb{R}$ be the linear real-valued function with domain $E$ where

$$
d(\tau)=(i+\lambda \tau) \frac{1}{\mu}+\left(\alpha_{k}-\tau\right)
$$

Suppose $D \in \mathbb{R}$. We claim for the followings:

1. Given $\rho \leq 1$. If $d(0) \leq D$ is satisfied, then $d(\tau) \leq D$ will be satisfied $\forall \tau \in E$.

2. Similarly, given $\rho>1$. If $d\left(\alpha_{k}\right) \leq D$ is satisfied, then $d(\tau) \leq D$ will be satisfied $\forall \tau \in E$.

Proof: See Appendix A about the mathematical proof for the Theorem 1.

\subsection{Battery Lifetime Expiry}

Once we can guarantee that the buffer overflow probability and the delay violation, the last criterion to check will be the battery lifetime. We want to make sure the power is sufficient to extend the sleeping mode by at least a period of , and the mobile station still has enough resources to perform the transmission to obtain all the packets coming to the buffer during the period of time.

Our objective is to verify the energy level sufficiency calculation. We will make use of the function derived for the delay violation. Similarly, suppose $\mu^{\prime}$ is the energy consumption rate. Then $\left[\alpha_{k} \lambda+i\right] \frac{1}{\mu^{\prime}}$ will be the total energy required to transmit $\alpha_{k} \lambda+i$ numbers of packets. If we subtract the energy required for sleeping and switching, then we have the following criteria:

$$
\left[\alpha_{k} \lambda+i\right] \frac{1}{\mu^{\prime}} \leq E_{\text {remain }}-\alpha_{k}^{\prime}
$$

where $\alpha_{k}^{\prime}=E_{\text {sleep }}+E_{S W}$.

\subsection{WAKSLP_DECISION Algorithm}

Based on the three defined criteria above, we propose a real-time heuristic algorithm called WAKSLP_DECISION Algorithm (refer to Algorithm 1) for a mobile device to make an appropriate decision for waking up to fully functional normal mode. We will explain the algorithm in details here. Algorithm 1 WAKSLP_DECISION Algorithm
Require: Traffic rate $\lambda$, service rate $\mu$, energy consumption
values $\left(\mu^{\prime}, \alpha_{k}^{\prime}\right), \alpha_{k}, B, i, D$, and $E_{\text {remain. }}$.
1: Run this algorithm during the listening interval when
the MOB_TRF-IND message is received. 2: loop

3: $\quad$ MOB_TRF-IND $(1=$ positive $/ 0=$ negative $)$ received 4: if MOB_WAK-IND $=1$ then

5: $\mathrm{C} 1$ :

$$
\sum_{n=L_{B S}-(i-1)}^{\infty} \frac{\left(\lambda \alpha_{k}\right)^{n} e^{-\lambda \alpha_{k}}}{n !} \leq B
$$

6: $\quad \mathrm{C} 2$ :

$$
i \cdot \frac{1}{\mu}+\alpha_{k} \leq D \text { for } \rho \leq 1
$$

$$
\left(\alpha_{k} \lambda+i\right) \cdot \frac{1}{\mu} \leq D \text { for } \rho>1
$$

7: $\quad$ C3:

$$
\left[\alpha_{k} \lambda+i\right] \frac{1}{\mu^{\prime}} \leq E_{\text {remain }}-\alpha_{k}^{\prime}
$$

8: $\quad$ if $(\mathrm{C} 1=$ false $)$ or $(\mathrm{C} 2=$ false $)$ or $(\mathrm{C} 3=$ false $)$ then

9: $\quad$ MOB_WAK-RSP $=1$

10: else

11:

12 :

13:

14:

$15:$

$16:$

17:

18:

19:

20:

21: end loop

22: Output:

23: MOB_WAK-RSP $=1$ \{wakeup fully and receive all packets in the buffer

24: MOB_WAK-RSP $=0\{$ continue to sleep with the next sleeping windows

The algorithm begins at each listening interval after the sleeping windows of $\alpha_{k}$. Based on the embedded information in the MOB_TRF-IND message, such as traffic indicator, $\lambda$ and $i$, the mobile station will decide whether it should wake up to full normal mode and begin to receive packets awaiting at the base station. Surely, the mobile station should follow the original standard and continue to sleep when negative indicator is received. In line 4 of the Algorithm 1, the mobile station makes the decision to run the algorithm or not. If the decision results in proceeding to the algorithm, the three criteria will be computed and evaluated. The three 
criteria proposed previously are found in line 5, 6 and 7 . In line 8 , the mobile station ensures that all three criteria are satisfied before continuing to sleep. Either one of them fails, the wakeup request will be set in line 9 . The sleep mode deactivation will be set within line 13 and line 16 . Once the decision is made, the algorithm will be terminated and the system will follow the decision made. Note that the computation cost is ignored as the speed of computation for the today available mobile CPU is substantially fast. Thus, it is expected that the algorithm will deliver the decision immediately.

\section{PERFORMANCE EVALUATION}

In this section, we evaluate the performance of the proposed scheme.

\subsection{Simulation Evaluation}

Our simulation model is built using the Matlab [10] simulator. To focus on the energy consumption efficiency of the algorithm and reduce the complexity of the simulation, we assume the simple point-to-point communication with one WiMAX base station and one mobile WiMAX station. We assume the traffic arrival pattern follows the Poisson distribution. In our simulation, we set the mobile station to perform the PSC of type II in the sleep mode operation with the $\alpha_{k}=9 \mathrm{~ms}$ as the constant sleeping window size.

As far as the setting for energy consumption parameters is concerned, we make use of an industrial manufactured mobile WiMAX chip [11] information so as to reflect the actual performance and enhance the validity of the simulation results. The energy consumption information is summarized in the Table 2. Assume the service rate $\mu$ is 0.5 packets per

Table 2: Power Consumption Information

\begin{tabular}{|c|c|}
\hline Working Mode & Power Consumption Level (mW) \\
\hline Normal & 280 \\
\hline Idle & 120 \\
\hline Listen & 120 \\
\hline Sleep & 10 \\
\hline Switching & $<10$ \\
\hline
\end{tabular}

ms. Packets are generated based on a given $\lambda$ and served according to the $\mu$. The total simulation time is 1000 seconds in each run. And we obtain the mean statistic of the energy consumption from 100 separate runs. All other values for the simulation parameters are tuned carefully in order to closely reflect the realistic system performance as well as to enhance the feasibility and reasonability of the simulation experiments.

\subsection{Simulation Results}

In this section, we present our simulation results to demonstrate the effectiveness and advantages of our proposed heuristic algorithm for power saving in the IEEE 802.16e standard. We compare our results mainly with the performance of the original standard due to the novelty of its methodology. Our objective is to illustrate the significance of this algorithm in saving the energy of mobile station by making a heuristic decision at the listening interval. Fig. 3 shows the total energy consumption of our algorithm compared with the standard. This consumption level is proportionally growing

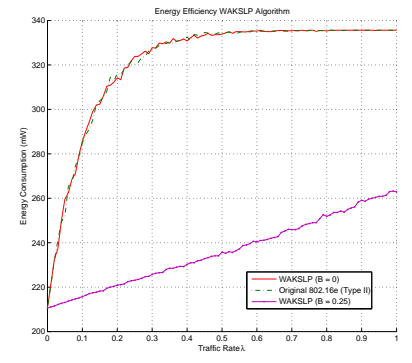

Figure 3: Comparison of WAKSLP Algorithm with Original Type II Sleep Mode $(B=0.25)$

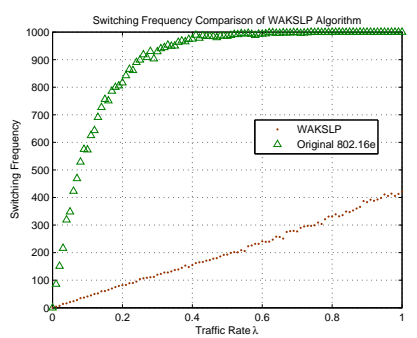

Figure 4: Switching frequency of WAKSLP Algorithm compared with Original Type II Sleep Mode

with the switching frequency between sleep mode and normal mode illustrated in the Fig. 4. In this simulation, the mobile station is willing to tolerate for $D<50 \mathrm{~ms}$ packet delay while ensuring the $B=0.25$ is the upper bound for the buffer overflow probability. Based on our algorithm, the station saves approximately $30 \%$ energy compared with the standard. Note that when $B$ becomes very stringent, meaning when buffer overflow is unacceptable at all, the algorithm tends to perform exactly the same as the original standard. This is indeed what we have expected because the algorithm will always make the decision to wake up at the listening interval whenever there is a positive traffic indicator. From this result, we can see that the worst case of our algorithm is to follow back to the original IEEE $802.16 \mathrm{e}$ standard for sleep mode operation. Thus, our algorithm has been shown to comply with the standard without altering it.

Fig. 5 shows an interesting observation about the parameter settings, and Fig. 6 illustrates the associated switching frequency. For both curves in Fig. 5 when $D=55 \mathrm{~ms}$ and

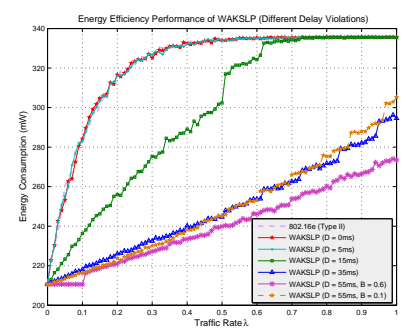

Figure 5: Energy efficiency of our proposed algorithm with the comparison of various delay requirements 


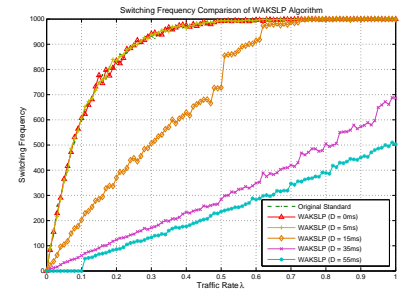

Figure 6: Switching Frequency of our proposed algorithm with the comparison of various delay requirements

$B=0.6$, we see a flat between $\lambda=0$ and 0.1 . In order to see the reason, let us compare this result with another one by setting the buffer overflow probability from 0.6 to 0.1. As we do so, the flat line disappears. This is a normal phenomenon because our decision making is based on three criteria. When either one is set to a very loose bound, the algorithm tends to enter to a deep sleep given that the energy is sufficient to sustain for a longer sleeping period. When the traffic rate is very low, it is common that the buffer overflow probability is proportionally low. If we set the upper bound high enough so that the actual probability will never violate our expectation, then the algorithm will decide not to wake up at all. Yet, this loose bound will be more likely to be violated when the traffic increases. Thus, we see the deep sleep is avoided when $\lambda$ increases.

\section{CONCLUSIONS}

In this paper, we propose a novel approach to provide with a promising solution to resolve the passive wake issue that minimizes the switching cost, and thus saves the overall energy consumption with the QoS guarantee support such as packet delay and packet loss. The results prove that it performs at least $25 \%$ better than the original standard and demonstrates its satisfactory. Therefore, we believe that this algorithm can serve as an add-on power saving feature to the original standard so that broadband wireless service can become robust and powerful to the linkage in the human communication.

\section{ACKNOWLEDGMENTS}

This work is supported by the RGC General Research Fund 620407. Also, the research was supported in part by grants from ITC HK ITP/023/08LP and the Foundation of Scientific and Technological Planning Project of Nansha District, Guangzhou, China.

\section{REFERENCES}

[1] IEEE 802.16-2004, "Part 16: Air Interface for Fixed Broadband Wireless Access Systems, Standard for Local and Metropolitan Area Networks", Octorber 2004.

[2] IEEE 802.16e-2006, "Part 16: Air Interface for Fixed and Mobile Broadband Wireless Access

Systems-Amendment for Physical and Medium Access Control Layers for Combined Fixed and Mobile Operation in Licensed Bands", February 2006.
[3] Yang Xiao, "Energy Saving Mechanism in the IEEE 802.16e Wireless MAN", IEEE Communication Letters, vol. 9, no.7, pp.595-597, July, 2005.

[4] Yang Xiao, "Performance Analysis of an Energy Saving Mechanism in the IEEE 802.16e Wireless MAN", Proceedings of IEEE CCNC 2006, Janurary, 2006.

[5] Lei Kong and Danny H. K. Tsang, "Performance Study of Power Saving Classes of Type I and II in IEEE 802.16e", Proc. of of the 31st IEEE Conference on Local Computer Networks, Tampa, Florida, US, November. 2006.

[6] Lei Kong and Danny H. K. Tsang, "Optimal Selection of Power Saving Classes in IEEE 802.16e", Proc. of IEEE Wireless Communications and Networking Conference ( WCNC 2007), Hong Kong, March, 2006.

[7] Yin Ge and G.S. Kuo, textquotedblleft An Efficient Sleep Mode Management Scheme in IEEE 802.16e Networks," in Proc of IEEE ICC'07, Glasgow, Scotland, 2007.

[8] M. Kim, M. Kang, and J. Choi, "Remaining Energy-Aware Power Management Mechanism in the 802.16e MAC," in Proc. IEEE CCNC 2008.

[9] Henk C. Tijms, A First course in Stochastic Models, John Wiley and Sons, Inc., New York, 2003.

[10] "Matlab," http://www.mathworks.com/

[11] Sequans Communications, "Datasheet: SQN1130 System-on-Chip (SOC) for WiMAX Mobile Stations"

\section{APPENDIX}

\section{A. PROOF OF THE EXPECTED DELAY VI- OLATION}

Proof. We will begin our proof by showing that $d$ is a continuous real-valued function on domain $E$. Let $p$ be a point where $p \in E$. Since for every $p$ in $E$, there exists a real value of $d(p)$. Hence, $d(p)$ exists for all $p \in E$ In addition, $\lim _{x \rightarrow p} d(p)$ exists and $\lim _{x \rightarrow p} d(x)=d(p)$ for all $p \in E$. By the definition of continuity, we have shown that $d$ is a continuous real-valued function on domain $E$.

Now, we are ready to proceed with our proof once we have shown that the function is a continuous real-valued function on domain $E$.

$$
\begin{aligned}
d(\tau) & =(i+\lambda \tau) \frac{1}{\mu}+\left(\alpha_{k}-\tau\right) \\
& =i \cdot \frac{1}{\mu}+\alpha_{k}+\left(\frac{\lambda}{\mu}-1\right) \tau \\
& =i \cdot \frac{1}{\mu}+\alpha_{k}+(\rho-1) \tau
\end{aligned}
$$

Now, if we take the derivative with respect to $\tau$, we have $d^{\prime}(\tau)=\rho-1$.

For $\rho<1, d^{\prime}(\tau)<0$. So the slope of the function is always negative. It implies that the initial end-point $d(0)$ is the absolute maxima. For $\rho>1, d^{\prime}(\tau)>0$. So the slope of the function is always positive. It implies that the end-point $d\left(\alpha_{k}\right)$ is the absolute maxima. So, our proof has shown that $d\left(\alpha_{k}\right)$ is the absolute maxima.

Thus, we can conclude that if $d(0) \leq D$ is satisfied, then $d(\tau) \leq D$ will be satisfied for $\forall \tau \in E$ given $\rho \leq 1$. Also, we can conclude that if $d\left(\alpha_{k}\right) \leq D$ is satisfied, then $d(\tau) \leq D$ will be satisfied for $\forall \tau \in E$ given $\rho>1$. 